\title{
Potential Applications of Soft-Computing Techniques for Human Socio-Cultural Behavior Modeling
}

\author{
Erman Çakit ${ }^{1 *}$, Waldemar Karwowski ${ }^{2}$ \\ ${ }^{1}$ Department of Industrial Engineering, Faculty of Engineering, Aksaray University, 68100 Aksaray, Turkey. \\ 2 Department of Industrial Engineering and Management Systems, University of Central Florida, Orlando, FL \\ 32816-2540 USA.
}

${ }^{*}$ Corresponding author. Tel.: +905368517595; email: ermancakit@aksaray.edu.tr Manuscript submitted November 9, 2015; accepted April 5, 2016.

doi: $10.17706 /$ jcp.12.4.284-290

\begin{abstract}
Human socio-cultural behavior (HSCB) modeling has received much attention in attempts to successfully understand the effects of social and cultural factors on human behavior. The principal aim of HSCB modeling is to better organize and control the human terrain during nonconventional warfare and other missions. The intent of this study is to review and classify the literature based on the applications of different kinds of data for modeling HSCB and highlight the soft-computing methodologies suitable for HSCB event/incident data that have been applied successfully to crime data.
\end{abstract}

Key words: Crime data, human socio-cultural behavior modeling, incident data, soft-computing.

\section{Human Socio-Cultural Behavior (HSCB) Modeling}

The U.S. military has made some adjustments to its force structure in recognizing the challenges of irregular warfare. The success of irregular warfare operations mostly depends on the safety of the civilian population, since the civilian population is at the center of irregular warfare (Fig. 1). When irregular warfare missions are involved, the human, social, and cultural elements should not be omitted to be successful. Bhattacharjee [1] outlined the application of a program called human socio-cultural behavior (HSCB) modeling to guide the U.S. military in achieving a better understanding of the different types of cultures encountered while operating in overseas countries [2]. The overarching aim of the HSCB Modeling is to enable Department of Defense (DoD) and the U.S. Government to better organize and control the human terrain during nonconventional warfare and other missions [3].

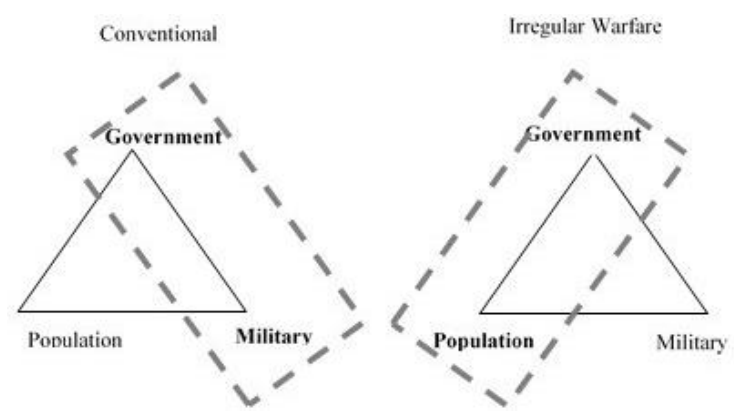

Fig. 1. Contrasting conventional and irregular warfare [4].

HSCB models are attracting much attention with regard to current and future operational requirements to 
be successful in understanding the effects of social and cultural factors on human behavior [5]-[7]. HSCB models are formed in order to understand the behavior and structure of organizational units at the macro level (economies, politics, and socio-cultural regions) and at the micro level (terrorist networks, tribes, military units) [8].

One area of research by computational social scientists involves understanding how observations of human behavior could be used to develop scientifically based models of HSCB events [9]. HSCB models are complex systems and require computational modeling and simulation techniques to handle this complexity. A number of different approaches to modeling data have been developed for this purpose; however, none of them has been widely accepted yet. In this framework, this study aims to i) review and classify the literature based on the applications with different kinds of data for modeling human socio-cultural behavior and ii) highlight the possible soft-computing techniques which can be suitable for HSCB event/incident data that have been applied successfully to the crime data.

\section{Challenges and General Modeling Approaches}

Using HSCB modeling to predict actions or reactions to events is a highly complex problem with many challenges. Studies in this area emerge from data-driven, statistical approaches where the modeler empirically derives the HSCB model from patterns identified in the data [10]. Trying to solve problems related to social science data represents the various difficulties encountered, and some of these challenges have been highlighted by other researchers. For instance, Numrich and Tolk [11] summarized these challenges: lack of common vocabulary, variations in modeling approaches, and data acquisition. Under this standpoint, some basic approaches are discussed and respective solutions are proposed in recent studies; a study by Schmorrow et al. [12] emphasized the challenge of leveraging modeling and simulation (M\&S) for HSCB to highlight the difficulty in understanding which M\&S tools are actually useful and when and how best to use M\&S tools within different complexity levels. Recently, Hahn [13] highlighted the challenges in verification and validation of HSCB models by reviewing the literature. The author concluded that empirically-based models are not often applicable for validation of HSCB models.

One of the solutions to the challenges was discussed in a study by Numrich [14] that aimed to deconstruct the problem of fitting HSCB data simulations into several steps, starting with the vocabularies in which they begin to express the problem space and concluding with a brief review of two architectures. The author concluded that the potential use for unifying architectures is to provide researchers and practitioners an environment in which to explore. For similar purposes, Tolk et al. [15] summarized the position of papers by inviting five internationally recognized people in the field of HSCB to get their ideas for the argument on methodological approaches to meet the difficulties in HSCB modeling. The main observation was called HSCB as a conceptual challenge that needs to be addressed on the modeling level.

In summary, there are several challenges with HSCB modeling, particularly with regard to handling complexity, uncertainty, etc. All the challenges illustrated by these authors must be understood; and researchers should meet specific modeling requirements before proceeding to apply various methodologies in the HSCB field. The use of soft computing techniques with HSCB can mitigate these challenges. A number of different approaches including geographical based models and agent-based approaches to model HSCB data are developed in the next sections; however, none has been accepted widely.

\subsection{Geographic Concentration of Terrorist Attacks}

The understanding of terrorism and counterterrorism must begin with a solid grasp of the historical patterns of terrorist incidents. For the purpose of understanding these patterns, geographically-oriented modeling should be performed. Since terrorist attacks are not random in space and time, patterns do exist. It is possible to discover representative patterns in adverse activity or behavior over time and space by 
analyzing geospatial intelligence on reported incidents, as stated by the Director of National Intelligence, Open Source Center [16].

The attacks against the United States (U.S.) on September 11th, 2001 dramatically increased the number of studies on terrorism and other related events. This change, named as a "second wave of terrorism research," encouraged new and innovative research approaches from many disciplines, including geographically-oriented studies [17]. Therefore, the applications of geographically-oriented modeling, in the domain of terrorism, is a relatively recent trend, and the work of spatial and temporal analysis of terrorist attacks is becoming increasingly important in the literature. Several studies have employed spatial and temporal analysis to analyze terrorism events.

The Open Source Center study of terrorism in Afghanistan highlighted various types of analysis that include spatial patterns and a distribution of incidents [18]. A study by LaFree et al. [19] on spatial and temporal patterns of all terrorist incidents (from 1970 to 2007) attributed to the ETA (a Spanish separatist group) concluded that incidents were mainly cumulated in the four main provinces in Spain, and $40 \%$ of all incidents in a given province were shortly followed by another incident in the same province. The similar pattern attributed to ETA is seen in another study by Behlendorf et al. [20] that concluded high geographic concentrations for incidents by province not only by ETA in Spain but also by the FMLN terrorist organization in El Salvador.

Berrebi and Lakdawalla [21] considered how terrorists sought targets and focused on the spatial and temporal determinants of terrorism in Israel between 1949 and 2004. Based on the analysis, they found that space and time are necessary to describe the patterns of terrorism in Israel. Very similar spatial relationships were found by Webb and Cutter [22] for terrorist incidents with respect to space and time in the United States over the years 1970 through 2004. In this paper, the authors highlighted several aspects of terrorist incidents by applying spatial statistics. Likewise, Johnson and Braithwaite [23] studied space-time clusters of improvised explosive device (IED) and non-IED attacks in Iraq from January to June of 2005.

Several studies showing how spatial models and GIS can be integrated with traditional statistical approaches useful for the immediate prevention, evacuation, and rescue efforts in the case of a terrorist attack. A study by Siebeneck et al. [24] used historical data of terrorist incidents in Iraq from 2004 to 2006 to develop a series of analyses to understand terrorist activity spaces and counter-terrorist actions. They applied several methodologies such as clustering analysis, spatial and temporal statistics, and GIS to detect and understand patterns. Brown et al. [25] highlighted a specific type of event (suicide bombings) to represent the difficulties in understanding and preventing terrorist attacks. They proposed a fusion model that integrates spatial likelihood modeling of environmental characteristics and logistic regression modeling of demographic features, and concluded that the fusion model performs better than other methods such as kernel density estimation methods.

\section{Prediction and Classification Models Using Crime and Terrorism-Based Dataset}

Techniques applied for detecting, monitoring, and estimating spatial patterns for crime analyses may also be beneficial in conducting studies of terrorist incident data. This is due to the fact that terrorist incidents are crimes, and thus, target selection for both incident and crime data are not random in space and time. In the specific case of crime prediction, semantic data for identifying the incidents is highly acceptable, as it is necessary to support decision-making processes and, in general, to prevent and correct policies [26].

Liu and Brown [27] applied point-pattern methods of geography to forecasting as one of the first applications in literature. This methodology has been described as a newly created space-time prediction model for crime points. They conclude that their model outperforms the best of current "hot spot" methods. Similarly, Gorr et al. [28] studied monthly crime data from 1991 to 1998 . They compared forecast 
accuracy of naive methods widely used by police and they found the most accurate forecast model to be the Holt exponential smoothing approach for precinct-level crime series. Polat [29] generated a model that predicts crime patterns by applying a time series forecasting approach with the help of GIS. The proposed model provided information for police departments in both space and time.

As a highly related paper to the prediction approach among terrorist based data, Reed et al. [30] aimed at demonstrating an argument that a statistical awareness of terrorists' behavior could be considered for estimating future behavior patterns. They applied a time-correlation based prediction approach and determined trends in the behavior of terrorists. They concluded that these trends could be adopted for predicting attacks in the future and that the trends might help for allocating more aid and people to the risky areas for possible terrorist attacks.

In spatial data in clustering, different kinds of clustering algorithms have been applied, "including spatial clustering (clustering of spatial points), regionalization (clustering with geographic contiguity constraints) and point pattern analysis (hot-spot detection with spatial scan statistics) and the use of many of these techniques for hot-spot detection is relatively problematic for several reasons, including the relatively arbitrary definition of the number of clusters to be included and the procedures applied to draw hot-spot boundaries" [31]. For instance, Kumar and Chandrasekar [26] applied a spatial-temporal prediction model to predict the criminal activity behavior in a specific district by applying a crime-based classification approach.

In the next section, we briefly describe the soft-computing concepts and review the literature relevant to its applications in crime and incident data. We then conclude our study and discuss the future work.

\section{Soft-Computing Modeling and Its Applications}

In this section, a short review is attempted to present the applications of soft-computing techniques, depicting the estimation and classification of crime and incident data. Recently, Çakit et al. [5] investigated the relationship between adverse events and infrastructure development investments in an active war theater by using soft computing techniques including fuzzy inference systems (FIS), artificial neural networks (ANNs), and adaptive neuro-fuzzy inference systems (ANFIS). They demonstrated that ANN, FIS, and ANFIS are useful modeling techniques for predicting the number of adverse events based on historical development or economic project data. It was concluded that the importance of infrastructure development projects varied based on the specific regions and time period.

For the same purpose, Cakit and Karwowski [6] investigated the relationship between adverse events and infrastructure development investments by estimating the number of adverse events in an active war theater based on the allocation of infrastructure development projects utilizing a fuzzy inference systems (FIS) approach. It was concluded that the FIS is a useful modeling approach that can be applied under scenario-based conditions to support decision makers in analyzing historical economic data on how allocation of regional infrastructure development funds can best help reducing the onset of adverse events in an active war theater.

Minu et al. [32] analyzed the time series of the number of terrorist attacks in the world measured on a monthly basis from 1968 to 2007. They concluded that wavelet neural networks provide the best model for analyzing terrorist attack time series, as opposed to other existing methods. Elkosantini and Gien [33] proposed a model that has two phases; the first one represented human behavior in a physiological and behavioral aspect, the second one integrated the sociological aspect. They applied fuzzy sets and fuzzy inference systems to describe the model and noted that the described model can be implemented in an agent-based approach. Corcoran et al. [34] demonstrated the training of artificial neural networks by applying GIS based criminal data to simplify predictive modeling. 
When there is a massive number of event-points, the traditional density approaches are not appropriate to determine hot-spots because of high computational complexity; then the application of cluster-based approaches is more useful: it is obvious that the clusters include similar datasets and the degree of affiliation is not strong between different data clusters [35]. For instance, Grubesic [36] presented an empirical analysis on the benefits of fuzzy cluster analysis for crime hot-spot detection. The results show that fuzzy clustering is a useful approach for dealing with intermediate points and spatial outliers while comparing with other traditional approaches utilized in spatial applications.

A fuzzy clustering algorithm allows data points to be part of several clusters concurrently with different degrees of membership. Several studies have employed fuzzy based cluster algorithms to identify criminal hot-spots. Inyaem et al. [37] applied fuzzy inference systems (FISs) for event classification in the domain of adverse incident analysis. They presented a comparison of these frameworks of classification using FISs with structured and unstructured events, and a comparison of structured event frameworks of classification using FIS and adaptive neuro-fuzzy inference systems (ANFIS) in incident monitoring domain. They concluded ANFIS gives better performance than FIS for event classification. Another study by Di Martino et al. [38] implemented the extended fuzzy C-means (EFCM) method in a GIS environment developed with the tool ESRI/ARCGIS. They showed that the extended fuzzy C-means (EFCM) algorithm works better than the classical FCM algorithm: indeed it determines automatically the initial number of clusters, it prevents the problem of shifting the clusters with low density areas of data points in areas with higher density of such points, and it finds the cluster volume prototypes as hyperspheres.

\section{Concluding Remarks and Further Work}

In summary, this study represents the benefits of employing soft-computing approaches in decision-making under the study of HSCB modeling. For future studies, it would be a basic review for HSCB researchers and decision makers to expand on the toolset for the domain of HSCB. There are still a lot of unknowns about HSCB and the model will probably never be good at making exact predictions of what will happen. It is anticipated that by examining these soft-computing advantages, this study may increase attention to the soft-computing based studies in literature and their contributions to HSCB modeling. In this context, it is hoped that this study would give HSCB researchers new tools and ideas on how to approach HSCB models using soft-computing methodologies besides geographical and agent-based approaches.

\section{Acknowledgment}

This study was supported in part by Grant No. 1052339, Complex Systems Engineering for Rapid Computational Socio-Cultural Network Analysis, from the Office of Naval Research. The authors acknowledge the helpful guidance of ONR program management, and the contributions of the technical team.

\section{References}

[1] Bhattacharjee, Y. (2007). Cross-cultural research. Pentagon asks academics for help in understanding its enemies. Science, 316(5824), 534-535.

[2] Drapeau, M. D., \& Mignone, B. K. (2007). Culture, conflict, and climate. Science, 316(5831), 1564.

[3] HSCB Modeling Program. (2009). From http://www.dtic.mil/biosys/docs/HSCB-news-spring-2009.pdf

[4] IWJOC. (2007). Irregular warfare joint operating concept. Retrieved 11 September 2007, from http://www.fas.org/irp/doddir/dod/iw-joc.pdf

[5] Çakıt, E., Karwowski, W., Bozkurt, H., Ahram, T., Thompson, W., Mikusinski, P., \& Lee, G. (2014). Investigating the relationship between adverse events and infrastructure development in an active war theater using soft computing techniques. Applied Soft Computing, 25, 204-214. 
[6] Cakit, E., \& Karwowski, W. (2015). Assessing the relationship between economic factors and adverse events in an active war theater using fuzzy inference system approach. International Journal of Machine Learning and Computing, 5(3), 252-257.

[7] Çakıt, E., \& Karwowski, W. (2015). Understanding Patterns of Infrastructure Development in the Active War Theater of Afghanistan over the Period 2002-2010. Procedia Manufacturing, 3, 3876-3882.

[8] Stanton, J. (2007, June). Evolutionary cognitive neuroscience: Dual use discipline for understanding \& managing complexity and altering warfare. Proceedings of International Studies Association Conference.

[9] Schmorrow, D., \& Nicholson, D. (2010). Advances in Cross-Cultural Decision Making, 38, 374-384.

[10] Van Hemel, S. B., MacMillan, J., \& Zacharias, G. L. (2008). Behavioral Modeling and Simulation: From Individuals to Societies, National Academies Press.

[11] Numrich, S. K., \& Tolk, A. (2010). Challenges for human, social, cultural, and behavioral modeling. SCS M\&S Magazine, 1(1), 1-9.

[12] Schmorrow, D., Klein, G. L., Foster, R., Boiney, J., Biggerstaff, S., Garvey, P. R., Koehler, M., \& Costa, B. (2009). Applied use of socio-cultural behavior modeling and simulation: An emerging challenge for C2. Ft. Belvoir: Defense Technical Information Center.

[13] Hahn, H. A. (2013). The conundrum of verification and validation of social science-based models. Procedia Computer Science, 16, 878-887.

[14] Numrick, S. K. (2010). Introduction to architectures: HSCB Information - what it is and how it fits (or doesn't fit). Proceedings of RTO Human Factors and Medicine Panel (HFM) Symposium, Netherlands.

[15] Tolk, A., Huiskamp, W., Schaub, H., Davis, P. K., Klein, G. L., \& Wall, J. A. (2010, December). Towards methodological approaches to meet the challenges of human, social, cultural, and behavioral (HSCB) modeling. Proceedings of the Winter Simulation Conference (pp. 912-924).

[16] Federation of American Scientists. (2009). 'A GEOINT analysis of terrorism in Afghanistan.' Secrecy news. Retrieved May 3, 2012 from http://www.fas.org/blog/secrecy/2009/05/05

[17] Pape, R. A. (2009). Introduction: What is new about research on terrorism. Security Studies, 18(4), 643-650.

[18] Open Source Center (OSC). (2009, April 30). Afghanistan-geospatial analysis reveal patterns in terrorist incidents 2004-2008. Retrieved May 3, 2012, from http://www.fas.org/irp/dni/osc/afghan-geospat.pdf

[19] LaFree, G., Dugan, L., Xie, M., \& Singh, P. (2012). Spatial and temporal patterns of terrorist attacks by ETA 1970 to 2007. Journal of Quantitative Criminology, 28(1), 7-29.

[20] Behlendorf, B., LaFree, G., \& Legault, R. (2012). Predicting microcycles of terrorist violence: Evidence from FMLN and ETA. Journal of Quantitative Criminology, 28, 49-75.

[21] Berrebi, C., \& Lakdawalla, D. (2007). How does terrorism risk vary across space and time? An analysis based on the Israeli experience. Defence and Peace Economics, 18(2), 113-131.

[22] Webb, J. J., \& Cutter, S. L. (2009). The geography of US terrorist incidents, 1970-2004. Terrorism and Political Violence, 21(3), 428-449.

[23] Johnson, S. D., \& Braithwaite, A. (2009). Spatio-temporal modeling of insurgency in Iraq. In J. D. Freilich, \& G. R. Newman, (Eds.), Reducing Terrorism through Situational Crime Prevention: Crime Prevention Studies, 25, 9-32, Monsey, NY: Criminal Justice Press.

[24] Siebeneck, L. K., Medina, R. M., Yamada, I., \& Hepner, G. F. (2009). Spatial and temporal analyses of terrorist incidents in Iraq, 2004-2006. Studies in Conflict \& Terrorism, 32(7), 591-610.

[25] Brown, D., Dalton, J., \& Hoyle, H. (2004). Spatial forecast methods for terrorist events in urban environments. Lecture Notes in Computer Science, 3073, 426-435.

[26] Kumar, M. V., \& Chandrasekar, C. (2011). Spatial clustering simulation on analysis of spatial temporal crime hotspot for predicting crime activities. International Journal of Computer Science and Information Technologies, 2(6), 2864-2867.

[27] Liu, H., \& Brown, D. E. (2003). Criminal incident prediction using a point-pattern-based density model. International journal of forecasting, 19(4), 603-622. 
[28] Gorr, W., Olligschlaeger, A., \& Thompson, Y. (2003). Short-term forecasting of crime. International Journal of Forecasting, 19(4), 579-594.

[29] Polat, E. (2007). Spatio-Temporal Crime Prediction Model Based on Analysis of Crime Clusters, Doctoral dissertation, Middle East Technical University, Turkey.

[30] Reed, G. S., Colley, W. N., \& Aviles, S. M. (2013). Analyzing behavior signatures for terrorist attack forecasting. The Journal of Defense Modeling and Simulation: Applications, Methodology, Technology, 10(2), 203-213.

[31] López-Caloca A. A., \& Reyes, C. (2012). Fuzzy Modeling of Geospatial Patterns, Fuzzy Logic-Emerging Technologies and Applications. From http://www.intechopen.com/books/fuzzy-logic-emerging-technologies-and-applications/fuzzy-modeli ng-of-spatial-patterns

[32] Minu, K. K., Lineesh, M. C., \& John, C. J. (2010). Wavelet neural networks for nonlinear time series analysis. Applied Mathematical Sciences, 4(50), 2485-2495.

[33] Elkosantini, S., \& Gien, D. (2007). Human behavior and social network simulation: Fuzzy sets/logic and agents-based approach. Proceedings of Agent-Directed Simulation, Part of the ACM/SCS Spring Simulation Multiconference (pp. 102-109).

[34] Corcoran, J. J., Wilson, I. D., \& Ware, J. A. (2003). Predicting the geo-temporal variations of crime and disorder. International Journal of Forecasting, 19(4), 623-634.

[35] Di Martino, F., \& Sessa, S. (2009). Implementation of the extended fuzzy C-means algorithm in geographic information systems. Journal of Uncertain Systems, 3(4), 298-306.

[36] Grubesic, T. H. (2006). On the application of fuzzy clustering for crime hot spot detection. Journal of Quantitative Criminology, 22(1), 77-105.

[37] Inyaem, U., Haruechaiyasak, C., Meesad, P., \& Tran, D. (2010). Terrorism event classification using fuzzy inference systems. International Journal of Computer Science and Information Security, 7(3).

[38] Di Martino, F., Loia, V., \& Sessa, S. (2008). Extended fuzzy C-means clustering algorithm for hotspot events in spatial analysis. International Journal of Hybrid Intelligent Systems, 5(1), 31-44.

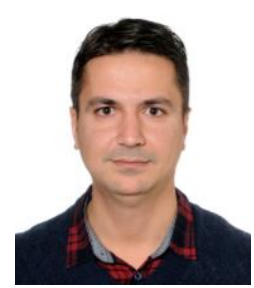

Erman Cakit received his Ph.D. in industrial engineering from the University of Central Florida, Orlando, USA, in 2013. He is currently working as an assistant professor at the Department of Industrial Engineering, Aksaray University, Turkey. His research interests include applications of human factors/ergonomics, cross cultural decision making, human social culture behavior (HSCB) modeling using soft computing, applied statistics, and geographic information systems. He is a member of IACSIT, SCIEI and IEEE Computational Intelligence Society.

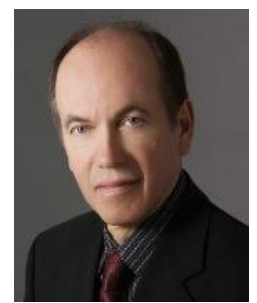

Waldemar Karwowski is a professor and chairman in the Department of Industrial Engineering and Management Systems and executive director in Institute for Advanced Systems Engineering, University of Central Florida, Orlando, USA. He held an M.S. in 1978 in production engineering and management from the Technical University of Wroclaw, Poland, and a Ph.D. in 1982 in industrial engineering from Texas Tech University. He was awarded a D.Sc. (dr habil) degree in management science by the State Institute for Organization and Management in Industry, Poland in 2004. He was a past president of the International Ergonomics Association (2000-2003), and past president of the Human Factors and Ergonomics Society in 2007. He served on the Committee on Human Factors/Human Systems Integration, National Research Council, the National Academies, USA (2007-2011). He is the author or coauthor of over 400 scientific publications which focus on occupational ergonomics, human systems integration, human-computer interaction, neuro-fuzzy modeling, nonlinear dynamics of human performance, and neuroergonomics. He is a member of IEEE/Systems, Man \& Cybernetics Society and Fellow Member of the Human Factors and Ergonomics Society. 